\title{
Gene expression of $O$-GIcNAc cycling enzymes in human breast cancers
}

\author{
Anna Krześlak · Ewa Forma • Magdalena Bernaciak • \\ Hanna Romanowicz $\cdot$ Magdalena Bryś
}

Received: 19 January 2011/Accepted: 21 April 2011/Published online: 13 May 2011

(C) The Author(s) 2011. This article is published with open access at Springerlink.com

\begin{abstract}
O$-GlcNAcylation is an abundant, dynamic, and inducible posttranslational modification in which single $\beta$ - $N$-acetylglucosamine residues are attached by $O$ glycosidic linkage to serine or treonine residues. It is suggested that abnormally regulated $O$-GlcNAcylation may contribute to the pathology of cancer. Cycling of $O$ GlcNAc residues on intracellular proteins is controlled by two enzymes, $O$-GlcNAc transferease (OGT), which catalyses the addition of $O$-GlcNAc residues and nucleocytoplasmic $\beta$ - $N$-acetylglucosaminidase ( $O$-GlcNAcase; encoded by MGEA5 gene), an enzyme involved in the removal of $O$-GlcNAc. In this study, relationship between the mRNA expressions of genes coding $O$-GlcNAc cycling enzymes in breast ductal carcinomas and clinicopathological parameters were analyzed. The results showed that poorly differentiated tumors (grade II and III) had significantly higher $O G T$ expression than grade I tumors. Contrary, MGEA5 transcript levels were significantly lower in grade II and III in comparison with grade I tumors. The Spearman rank correlation showed the expressions of $O G T$ and MGEA5 in breast cancer was negatively correlated $(r=-0.430, P=0.0002)$. Lymph node metastasis status was significantly associated with decreased MGEA5 mRNA expression. This result suggests that elevation in $O$-GlcNAc modification
\end{abstract}

A. Krześlak · E. Forma · M. Bernaciak · M. Bryś $(\bowtie)$

Department of Cytobiochemistry, University of Lodz,

Pomorska 141/143, 90-236 Łódź, Poland

e-mail: zreg@biol.uni.lodz.pl

H. Romanowicz

Department of Clinical Pathomorphology, Polish Mother's

Memorial Hospital Research Institute, Łódź,

Rzgowska 281/289, 93-338 Łódź, Poland of proteins may be implicated in breast tumor progression and metastasis.

Keywords Breast cancer $\cdot O$-GlcNAc $\cdot M G E A 5 \cdot O G T$

\section{Introduction}

Multiple nucleocytoplasmic proteins, including transcription factors, cytoskeletal proteins, oncogenes, and kinases, are modified with $\beta$ - $N$-acetylglucosamine residues attached by $O$ - $\beta$-glycosidic linkage $(O$-GlcNAc) [1-3]. $O$ GlcNAc is a dynamic and inducible posttranslational modification involved in protein regulation in a manner similar to phosphorylation. $O$-GlcNAc level on many proteins rapidly changes in response to different physiological stimuli, such as hormones, growth factors, and mitogens [4]. There is a relationship between $O$-GlcNAcylation and phosphorylation. All $O$-GlcNAc modified proteins are also phosphoproteins, and sometimes $O$ GlcNAc and $O$-phosphate moieties can compete for a binding site or alternatively $O$-GlcNAcylation and phosphorylation can compete via steric hindrance when the substrate modification sites are within 10 aminoacids from each other [5]. $O$-GlcNAc can modulate protein function by regulating protein activity, protein-protein interaction, localization, and protein degradation [6].

Unlike phosphorylation, which is determined by hundreds of kinases and phosphatases, cycling of $O$-GlcNAc residues on intracellular proteins is controlled only by two highly conserved enzymes. $O$-GlcNAc transferase (OGT) catalyses the addition of $O$-GlcNAc to proteins, and nucleocytoplasmic $\beta$ - $N$-acetylglucosaminidase (O-GlcNAcase) is an enzyme involved in the removal of $O$-GlcNAc. The cloned sequence of $O$-GlcNAcase was found to be 
identical to that of MGEA5 (meningioma-expressed antigen 5), which had been identified genetically in human meningiomas $[7,8]$.

Abnormally regulated $O$-GlcNAc has been found to be implicated in diabetes and neurodegenerative diseases [9]. It is also strongly suggested that alterations in $O$-GlcNAc cycling may contribute to the pathology of other human diseases, including cancers $[10,11]$. Overexpression of OGT causes defective cytokinesis increasing polyploidy of cells, a feature common to many cancer cells [12]. $O$ GlcNAc is present in many transcription and cell cycle regulatory proteins. The protooncogene $\mathrm{c}-\mathrm{Myc}$, which regulates transcription of genes involved in cell proliferation, apoptosis, and metabolism is modified by $O$-GlcNAc at Thr58. This treonine residue within transactivation domain is also a GSK3 $\beta$ phosphorylation site. Phosphorylation of this site reduces protein stability and leads to c-Myc degradation and a reduction in c-Myc target gene expression. By contrast, an $O$-GlcNAc residue at Thr58 can block phosphorylation and potentially stabilize the protein. Frequent mutation of this site is found in human lymphomas, demonstrating the importance of this site in tumor progression [13]. The important tumor suppressor p53, which is mutant or dysregulated in nearly all cancers bears $O$-GlcNAc at Ser149. The stability of p53 is also tightly reduced by phosphorylation. $O$-GlcNAcylation of Ser149 reduces phosphorylation at Thr155, which subsequently blocks the interaction with Mdm2, an E3 ubiquitin ligase. Thus, increased $O$-GlcNAcylation of p53 at Ser149 results in decreased p53 ubiquitination and stabilizes the p53 protein [14].

$O$-GlcNAc and phosphorylation crosstalk can be more complicated than only competition between the two modifications on the same proteins. Cell has to keep a right balance between these two modifications to survive and develop normally. Alterations in $O$-GlcNAcylation play a role in tumorigenesis and progression of cancers. It has been shown that breast cancer cell lines contain elevated $O$ GlcNAcylation and OGT mRNA levels compared with normal cell lines [15]. Yu and coworkers have demonstrated that $O$-GlcNAcylation level of lung, colon, and breast cancer tissue is significantly increased compared with that in corresponding adjacent tissues $[16,17]$. The intracellular $O$-GlcNAcylation has been found to be associated also with the pathogenesis of chronic lymphatic leukemia (CLL) [18]. However, aggressive clinical behavior of CLL cells was found to correlate with lower $O$ GlcNAc level.

In this study, we analyzed $O G T$ and MGEA5 mRNAs expression levels in breast ductal carcinomas and the relationship between their expression and clinicopathological parameters such as tumor grade, lymph node metastasis status or estrogen, and progesterone status.

\section{Materials and methods}

Patients and samples

The studied material was obtained from Department of Clinical Pathomorphology Polish Mother's Memorial Hospital, Research Institute, Łódź, Poland and comprised samples of 70 breast ductal carcinomas. The mean patient age was 57 (range 39-72). Tumor grade was assessed according to Bloom-Richardson criteria (21 tumors were of grade I, 30 of grade II and 19 of grade III). Twenty-six tumor samples were classified as lymph node metastasis positive and 44 as lymph node negative. Both estrogen receptor (ER) and progesterone receptor (PR) expression was stated in 33 tumor samples. Twenty-one samples were negative for both receptors and 16 showed expression one of the two receptors. Tissue samples of 24 normal breasts were obtained from women undergoing radical mastectomy.

Total RNA extraction and cDNA synthesis

Total RNA was extracted using TRI Reagent (SigmaAldrich, USA) according to manufacturer's protocol. RNA was eluted in $20 \mu \mathrm{l}$ RNase-free water, quantified by spectrophotometry at $260 \mathrm{~nm}$ and stored at $-20^{\circ} \mathrm{C}$. RNA with a $260 / 280 \mathrm{~nm}$ ratio in range $1.8-2.0$ was considered high quality. First strand cDNAs were obtained by reverse transcription of $1 \mu \mathrm{g}$ of total RNA using RevertAid ${ }^{\mathrm{TM}}$ First strand cDNA synthesis kit (Fermentas International, Lithuania) following the manufacturer's protocol.

\section{Real-time quantitative PCR}

Real-time amplification of the cDNA was performed using TaqMan ${ }^{\circledR}$ Gene Expression Assay (Applied Biosystems) according to manufacturer's instruction. The fluorogenic, FAM labeled probes, and the sequence-specific primers for $O G T, M G E A 5$, and internal control GAPDH were obtained as inventoried assays (Hs00201970, Hs00269228 and Hs99999905, respectively). In samples with positive expression of target genes, the curve of fluorescence quantitative amplification showed an typical S-line, and in negative samples, the curve was an irregular wavy line. All positive samples had $\mathrm{Ct}$ (cycle threshold) $\leq 30$. If the $\mathrm{Ct}$ mean value was more than 40 , the sample was defined as negative in the expression of the target gene. The level of $O G T$ and MGEA5 mRNA in samples with positive expression was quantified by the $\Delta \mathrm{Ct}$ method. $\Delta \mathrm{Ct}$ (Ctgene-CtGAPDH) values were recalculated into relative copy number values (number of $O G T$ or MGEA5 mRNA copies per 1,000 copies of GAPDH mRNA). 
Statistical analysis

The statistical analyses were performed using the STATISTICA version 9.0 (StatSoft, Poland). Since levels of expression in breast cancer specimens did not show normal distribution (Kolmogorov-Smirnov test), the nonparametrical statistical tests (Mann-Whitney $U$ test, KruskalWallis test or the Spearaman rank correlation test) were applied.

The chi-square test was used to identify relationship between expression of $O G T$ or MGEA5 and clinicopathological parameters. A $P$ value $<0.05$ was considered as statistically significant.

\section{Results and discussion}

There are not many data available concerning the expression and activity of $O$-GlcNAc cycling enzymes in human cancers. Mi et al. [16] analyzed by immunohistochemistry the expressions of OGT and $O$-GlcNAcase in lung and colon cancers. They found that OGT expression was elevated in both lung and colon tumor tissues compared with their corresponding adjacent tissues. Elevated OGT mRNA expression had been found in breast cancer cell lines and breast invasive ductal carcinoma compared with normal breast cells and normal breast tissue [15]. One study evaluated the usefulness of MGEA5 expression status of breast cancer cells in revealing tumorigenesis. Dahl et al. [19] defined a set of candidate genes that are predominantly localized in the most frequently altered chromosomal regions known to be important in the pathogenesis of breast and ovarian cancers and then performed systematic characterization of these candidate genes by cDNA dot blot using cancer profiling array and real-time reverse transcription -PCR (RT-PCR) analysis. MGEA5 was one of the analyzed genes. Authors compared mRNA expression of MGEA5 in matched tumor/normal samples of 24 invasive ductal breast cancers. The results showed down-regulated expression of MGEA5 in tumor samples. Slawson et al. [20] investigated $O$-GlcNAcase activity using biochemical assay in 12 primary breast tumors compared with matched adjacent breast tissue. They demonstrated an increase in $O$-GlcNAcase activity in breast tumor tissue compared with normal tissue. However, because the data set was relatively small, there was no clear correlation with tumor grade or type. The results showing increased activity of $O$-GlcNAcase in breast cancer are not consistent with MGEA5 mRNA expression results.

The aim of our study was to establish whether there is any correlation between $O G T$ and MGEA5 expression and tumor grade, lymph node metastasis status or ER/PR status of human breast cancer. Levels of OGT and MGEA5
mRNAs were determined in tumor samples from 70 patients with breast ductal carcinomas and in 24 samples of normal breast tissue. In normal breast tissue, $O G T$ was expressed only in $66.7 \%$ of samples (16/24) and MGEA5 in $100 \%$ of samples (24/24). Positive expression of $O G T$ was found in 59 and MGEA5 in 57 of 70 carcinoma cases. The $O G T$ expression was most frequently observed in grade III tumors (94\%), while MGEA5 was most frequently expressed in grade I tumors $(95.2 \%)$ (Table 1). There was no statistically significant differences in MGEA5 expression frequency between grade I and grade II groups. However, the results showed a trend of poorly differentiated tumors (grade II and III) to be less frequently MGEA5 positive than well differentiated ones (grade I). The difference between grade I and III was statistically significant $(P=0.02)$. The expression level of $O G T$ mRNA was significantly lower in normal tissue compared with carcinoma tissue (Fig. 1). The expression of $O G T$ mRNA was significantly higher in grade II and III tumors than in grade I tumors $(P<0.05$, Fig. 1$)$. Contrary, MGEA5 transcript levels were significantly lower in grade II and III in comparison with grade I tumors. The results of Spearman rank correlation showed the expressions of $O G T$ and MGEA5 in breast cancers were negatively correlated $(r=-0.430$, $P=0.0002$ ). Lymph node metastasis status was significantly associated with decreased MGEA5 mRNA expression $(P<0.05)$ but not with $O G T$ mRNA expression. Both $O G T$ and MGEA5 expression profiles showed no significant differences in association with different estrogen and progesterone status (Fig. 1).

Our results showing decrease in MGEA5 and increase in $O G T$ expression in higher grade tumors are in agreement with earlier findings suggesting that increased $O$-GlcNAc modification may be implicated in breast tumor

Table 1 Expression of $O G T$ and MGEA5 in ductal breast carcinoma and normal breast tissue

\begin{tabular}{lll}
\hline Clinical characteristics & $O G T$ positive/total \% & $\begin{array}{l}\text { MGEA5 } \\
\text { positive/total } \%\end{array}$ \\
\hline $\begin{array}{l}\text { Normal tissue } \\
\text { Tumor grade }\end{array}$ & $24 / 24(100)$ \\
I & $16 / 24(66.7)$ & $20 / 21(95.2)$ \\
II & $17 / 21(80.9)$ & $24 / 30(80.0)$ \\
III & $24 / 30(80.0)$ & $13 / 19(68.4)$ \\
Lymph node metastasis & $18 / 19(94.7)$ & \\
No & $37 / 44(84.1)$ & $39 / 44(88.6)$ \\
Yes & $22 / 26(84.6)$ & $18 / 26(69.2)$ \\
$E R$ and $P R$ status & & \\
$E R+P R+$ & $28 / 33(84.8)$ & $26 / 33(78.8)$ \\
$E R+P R-/ E R-P R+$ & $13 / 16(81.3)$ & $15 / 16(93.7)$ \\
$E R-P R-$ & $18 / 21(85.7)$ & $16 / 21(76.2)$ \\
\hline
\end{tabular}



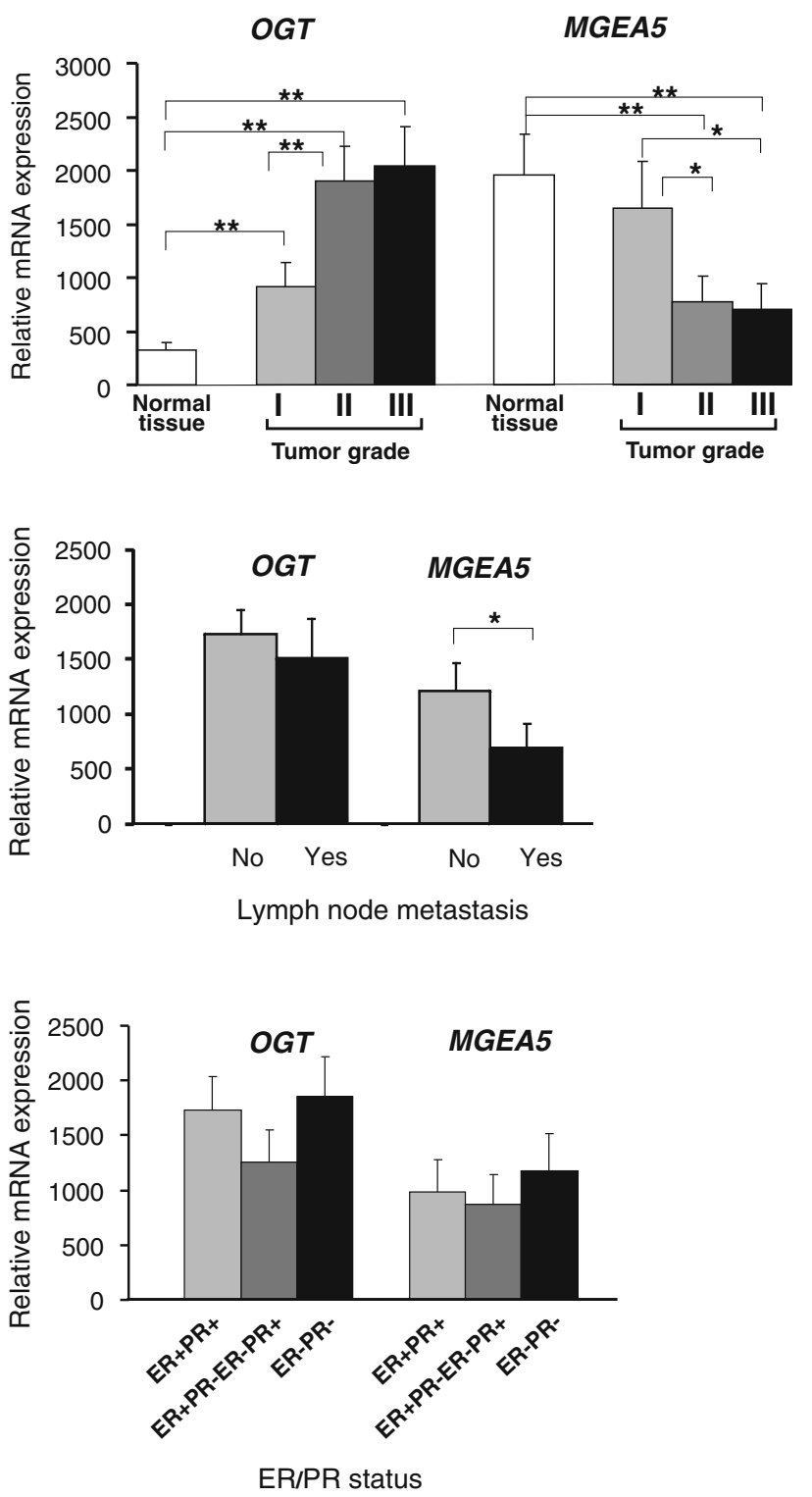

Fig. $1 O G T$ and MGEA5 mean mRNA expression level in ductal breast cancers; a comparison between subgroups with different tumor grade, lymph node metastasis status, and estrogen/progesterone status. Error bars represents standard error; $* P<0.05, * * P<0.01$, $* * * P<0.001$

progression and metastasis. Gu et al. [17] demonstrated that high $O$-GlcNAcylation enhanced the migration of breast cancer cells in vitro and lung metastasis in vivo. Moreover, Caldwell et al. [15] showed that reduction in $O$ GlcNAc level through RNA interference of OGT transferase in breast cancer cells lead to inhibition of tumor growth and is associated with decreased cancer cell invasion. Thus, the increase in $O$-GlcNAcylation seems to be beneficial to breast cancer cells. Negative correlation in expression of both enzymes may suggest that observed earlier abnormally high level of $O$-GlcNAcylation in breast cancer cells is not only an effect of increased level in
$O$-GlcNAc transferase but also depends on decreased level in $O$-GlcNAcase. We suggest that diagnosis, evaluation of metastasis potential and prognosis, would benefit from the combined detection of $O G T$ and MGEA5 expression.

The findings in this study suggest that $O$-GlcNAc cycling enzymes may be related to invasion and metastasis of ductal breast carcinoma and may become an important indicator for tumor progression. Further studies on $O$ GlcNAc cycling enzymes in breast cancer cells can provide insights into the molecular mechanism of disease progression and potentially new therapeutic targets.

Conflict of interest The authors declare that they have no conflict of interest.

Open Access This article is distributed under the terms of the Creative Commons Attribution Noncommercial License which permits any noncommercial use, distribution, and reproduction in any medium, provided the original author(s) and source are credited.

\section{References}

1. Zachara NE, Hart GW (2006) Cell signaling, the essential role of O-GlcNAc. Biochim Biophys Acta 1761:599-617

2. Hart GW, Housley MP, Slawson C (2007) Cycling of O-linked $\beta$ $\mathrm{N}$-acetylglucosamine on nucleocytoplasmic proteins. Nature 446:1017-1022

3. Özcan S, Andrali SS, Cantrell JEL (2010) Modulation of transcription factor function by O-GlcNAc modification. Biochim Biophys Acta 1799:353-364

4. Hu P, Shimoji S, Hart GW (2010) Site-specific interplay between O-GlcNAcylation and phosphorylation in cellular regulation. FEBS Lett 584:2526-2538

5. Butkinaree C, Park K, Hart GW (2010) O-linked beta-N-acetylglucosamine (O-GlcNAc): extensive crosstalk with phosphorylation to regulate signaling and transcription in response to nutrients and stress. Biochim Biophys Acta 1800:96-106

6. Zeidan Q, Hart GW (2010) The intersections between O-GlcNAcylation and phosphorylation: implications for multiple signaling pathways. J Cell Sci 123:13-22

7. Heckel D, Comtesse N, Brass N, Blin N, Zang KD, Meese E (1998) Novel immunogenic antigen homologous to hyaluronidase in meningioma. Hum Mol Genet 7:1859-1872

8. Comtesse N, Maldener E, Meese E (2001) Identification of a nuclear variant of MGEA5, a cytoplasmic hyaluronidase and a beta-N-acetylglucosaminidase. Biochem Biophys Res Commun 283:634-640

9. Lazarus BD, Love DC, Hanover JA (2009) O-GlcNAc cycling: implications for neurodegenerative disorders. Int J Biochem Cell Biol 41:2134-2146

10. Hanover JA, Krause MW, Love DC (2010) The hexosamine signaling pathway: O-GlcNAc cycling in feast or famine. Biochim Biophys Acta 1800:80-95

11. Slawson C, Copeland RJ, Hart GW (2010) O-GlcNAc signaling: a metabolic link between diabetes and cancer? Trends Biochem Sci 35:547-555

12. Slawson C, Zachara NE, Vosseller K, Cheung WD, Lane MD, Hart GW (2005) Perturbations in O-linked-beta-N-acetylglucosamine protein modification causes in severe defects in mitotic progression and cytokinesis. J Biol Chem 280:32944-32956 
13. Kamemura K, Hayes BK, Comer FI, Hart GW (2002) Dynamic interplay between O-glycosylation and O-phosphorylation of nucleocytoplasmic proteins: alternative glycosylation/phosphorylation of Thr58, a known mutational hot spot of c-myc in lymphomas, is regulated by mitogens. $\mathrm{J}$ Biol Chem 277:19229-19235

14. Yang WH, Kim JE, Nam HW, Ju JW, Kim HS, Kim YS, Cho JW (2006) Modification of p53 with O-linked N-acetylglucosamine regulates p53 activity and stability. Nat Cell Biol 8:1074-1083

15. Caldwell SA, Jackson SR, Shahriari KS, Lynch TP, Sethi G, Walker S, Vosseller K, Reginato MJ (2010) Nutrient sensor O-GlcNAc transferase regulates breast cancer tumorigenesis through targeting of the oncogenic transcription factor FoxM1. Oncogene 29:2831-2842

16. Mi W, Gu Y, Han C, Liu H, Fan Q, Zhang X, Cong Q, Yu W (2011) O-GlcNAcylation is a novel regulator of lung and colon cancer malignancy. Biochim Biophys Acta 1812:514-519
17. Gu Y, Mi W, Ge Y, Liu H, Fan Q, Han C, Yang J, Han F, Liu X, $\mathrm{Yu}$ W (2010) GlcNAcylation plays an essentials role in breast cancer metastasis. Cancer Res 70:6344-6351

18. Shi Y, Tomic J, Wen F, Shaha S, Bahlo A, Harrison R, Dennis JW, Williams R, Gross BJ, Walker S, Zuccolo J, Deans JP, Hart GW, Spaner DE (2010) Aberrant O-GlcNAcylation characterizes chronic lymphatic leukemia. Leukemia 12:1588-1598

19. Dahl E, Sadr-Nabavi A, Klopocki E et al (2005) Systematic identification and molecular characterization of genes differentially expressed in breast and ovarian cancer. J Pathol 205:21-28

20. Slawson C, Pidala I, Potter R (2001) Increased N-acetyl-betaglucosaminidase activity in primary breast carcinomas corresponds to a decrease in $\mathrm{N}$-acetylglucosamine containing proteins. Biochim Biophys Acta 1537:147-157 